
\title{
25 Research Suare \\ Effect of HO-1 modified BMMSCs on immune function of liver transplantation
}

Peng Li

Hubei University of Medicine

Yuyi Zhang ( $\nabla$ sharpfast@sina.com )

Hubei University of Medicine

Qiongxia Li

Hubei University of Medicine

Yubo Zhang

Wenzhou Medical University

\section{Research Article}

Keywords: HO-1囚BMMSCs $₫$ Liver transplantation $₫$ Regulatory $T$ cells $₫$ Treg $\rrbracket$

Posted Date: April 14th, 2021

DOI: https://doi.org/10.21203/rs.3.rs-388289/v1

License: (c) (1) This work is licensed under a Creative Commons Attribution 4.0 International License.

Read Full License

Version of Record: A version of this preprint was published at Scientific Reports on February 23rd, 2022.

See the published version at https://doi.org/10.1038/s41598-022-06141-7. 


\section{Abstract}

We examined whether HO-1could enhance the immunosuppressive effects of bone marrow mesenchymal stem cell $\mathbb{B} B M M S C s \bowtie$ on the rejection of transplanted liver allograft in rats. The rejection model of orthotopic liver transplantation in rats was established then BMMSCs orHO-1/BMMSCs were transfused via the portal vein.The histopathological changes of the transplanted liver and the change of regulatory $T$ cell囚Tregs natural killer cell囚NK囚and cytokines after transplantation were observed in different groups. The severe acute rejection model rats after liver transplantation on post operation day 10 showed in NS group. BMMSCs group showed strong protective effects on rejection within the first 10 days after transplantation. While HO-1/BMMSCs showed stronger effects than BMMSCs which provided enhanced

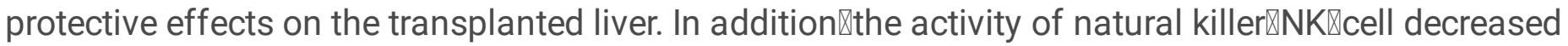

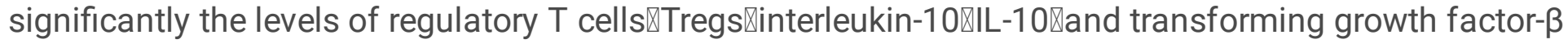

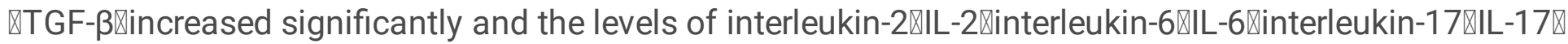

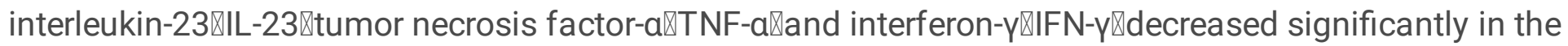
HO-1/BMMSCs group when compared with the BMMSCs group. Conclusions:HO-1/BMMSCs showed better immunosuppressive effects after transplantation. HO-1 could enhance the effects of BMMSCs inhibiting the immune response of acute rejection in orthotopic liver transplantation model of rats.

\section{Introduction}

Liver transplantation (LT) is an effective treatment for patients with liver failure and End-stage liver disease. However,the rejection of liver transplantation is obvious and there is no effective control means. The existing immunosuppressive treatments are not effective】and will bring some corresponding risks, such as malignant tumors, infections and high costs $\square$ which will seriously affect the long-term survival rate of liver transplant recipients and also restrict LT business.Development of Immune tolerance is a long-term $\llbracket$ non-responsive state of the recipient's immune system to the graft $\square$ and still has normal immune response to other foreign antigens. Therefore, how to induce a durable and drug-free immune tolerance is an urgent problem to be solved ${ }^{[1]}$.

Bone marrow mesenchymal stem cells(BMMSCs) have important application value in organ transplantation ${ }^{[2]}$. BMMSCs have low immunogenicity and have a certain effect on inhibiting T cellmediated immune rejection after organ transplantation ${ }^{[3-4]}$.It can secrete some immunosuppressive cytokines such as interleukin-10(IL-10),transforming growth factor- $\beta$ (TGF- $\beta$ ) )etc $\square$ as well as interfering with helper Tlymphocytes differentiation to induce immune tolerance. ${ }^{[5-6]}$ This is a promising treatment.However $\square$ it has also been reported that the activity of BMMSCs infusion alone in tissues is lower】and studies have shown that genetic engineering intervention to BMMSCs is a more effective method $^{[7]}$. Hemeoxygenase-1 ( $\left.\mathrm{HO}-1\right)$ is an immunomodulatory active factor involved in the regulation of immune tolerance after organ transplantation ${ }^{[8]}$. HO- 1 can be transfected into BMMSCs to enhance their immunomodulatory and antioxidant capacity while also prolonging their action time ${ }^{[9]}$. 
In this study,based on the simple application of BMMSCs to protect liver transplant rejection $\square$ the rat $\mathrm{HO}-1$ gene was transfected into BMMSCs to form HO-1/BMMSCs in vitro to study its effect in orthotopic rat liver transplantation. We willexplore whether $\mathrm{HO}-1$ can enhance regulatory effect of BMMSCs in transplant immunity and its mechanism of action.

\section{Materials And Methods}

\section{Materials}

DMEM/F12(1:1) medium (Hyclone, USA), fetal bovine serum (PAA囚Australia)『glutamate】

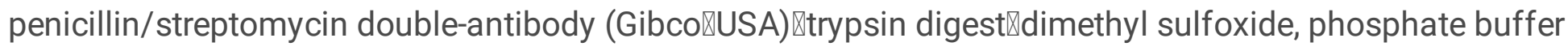

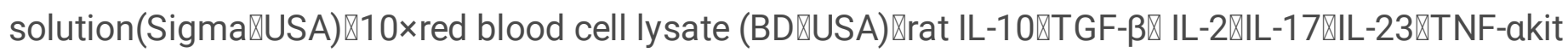

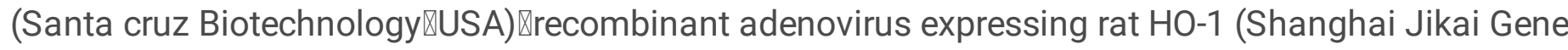
Chemical Technology Co.Ltd囚China). Leicac0269 surgical microscope (Leica microsystems AG》 Germany) \microsurgical instruments (Admiralty Medical Devices \China) \upright fluorescence

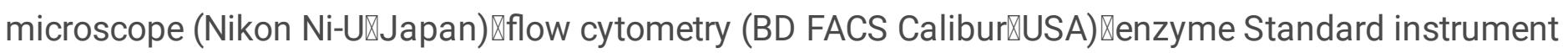
(Bio Tek Synergy 2هUSA).

\section{Animal experimental groups}

Experimental animals were purchased and maintained from our Experimental Animal Centerin the specific pathogen-free condition. The use and procedures to rats in this study was approved by the Animal Care and Ethics Committee of Hubei University of Medicine. SPF healthy male BN rats $\left(R T-1^{\mathrm{n}}\right)$ and male Lewis rats $\left(\mathrm{RT}-1^{1}\right) \otimes \mathrm{BN}$ rats weigh $180-200 \mathrm{~g} \otimes$ Lewis rats $200-220 \mathrm{~g}$. The animals were placed in the environment of light-darkness cycling every 12 hours and were fed adaptively for 7 days.BN rats were used as donors and Lewis rats were used as recipients to establish an allogeneic (Allo) acute rejection model. Rats were injected with normal saline(NS) or cells via the dorsal penile vein before operation. The experiment was divided into three groups $₫ N S$ group $\triangle B M M S C$ group and $\mathrm{HO}-1 / B M M S C$ group.Five rats were allocated to each group at $0 \otimes 1 \otimes 5 \otimes 7 \otimes 10$ day respectively. Experimental group was given $5 \times 10^{6} / \mathrm{ml}$ cells $(1 \mathrm{ml} / \mathrm{rat})$.Control group was given $\mathrm{NS}(1 \mathrm{ml} / \mathrm{rat})$. We observed the rats which were killed at 10 days after transplantation and collected peripheral venous blood and tissue samples. The rats follows the recommendations in the ARRIVE guidelines.

\section{The establishment of liver transplantation model}

The establishment of an animal model of orthotopic liver transplantation is the same as previous studies $^{[10]} .5 \times 10^{6} / \mathrm{ml}$ of HO-1/BMMSCs and BMMSCs were infused via portal vein when orperation.Postoperativecare:feeding in single cage after surgery and to drink freely after waking and to eat freely after 12 hours. Clean and disinfect the cage regularly and change the dressing to keep it clean.Observe the survival state of rats after transplantation and record the survival time of rats in each group. We observe the American Veterinary Medical Association (AVMA) Guidelines for the Euthanasia of Animals (2020) 


\section{Extraction凹culture and HO-1 transfection of BMMSCs}

The method of verification to BMMSCs is based on our previous research ${ }^{[10]}$. The expression of $\mathrm{HO}-1$ in HO-1/BMMSC was detected by immunocytochemical staining. The procedure was to place the sterilized cover slip on the bottom of a sterile Petri dish and take $5 \mathrm{ml}$ of adenovirus-loaded $\mathrm{HO}-1$ to the stained BMMSCs suspension(density $1 \times 10^{6} / \mathrm{ml}$ ) in the culture dish.After the cells were grown and fused $\$ rinsed it 3 times with PBS and 4\% paraformaldehyde treatment for 20 minutes.0.5\% Txiton X-100 for 20 minutes at room temperature『rinse $3 \% \mathrm{H}_{2} \mathrm{O}_{2}$ for 15 minutes at room temperature冈rinse $100 \mathrm{ml} / \mathrm{L}$ normal sheep serum blocking solution for 1 hour.Add 1:100 Rabbit anti mouse $\mathrm{HO}-1$ antibody on the slide $\varangle 4^{\circ} \mathrm{C}$ Fridge overnight after washing.After rinsing \add 1:400 PE-labeled Sheep anti-Rabbit immunofluorescence antibody and incubate in $37^{\circ} \mathrm{C}$ water bath for 1 hour in dark. Seal and observe the red fluorescence under fluorescence microscope after rinsing.

\section{Histopathological examination and acute rejection score}

The histopathological examination of each group was performed on the 10 days after operation. The histopathological changes were observed by optical microscope. According to the criteria of acute rejection $\nabla$ the degree of rejection at each time point of each group of rats was scored and statistical analysis was performed.Under the optical microscope $₫ 5$ fields were randomly selected to observe the pathological changes of the two groups of animals. The liver transplant rejection score was based on the Banff international standard formulated in 1997.

\section{Detection of NK cell activity in recipient}

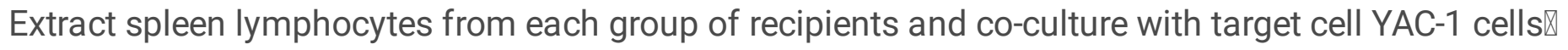
calculate the activity of NK cells by measuring the release amount of lactate dehydrogenase. Statistical analysis Differences between groups $₫ \mathrm{P} \otimes 0.05$

\section{Detection of immune-related cytokines and Tregs}

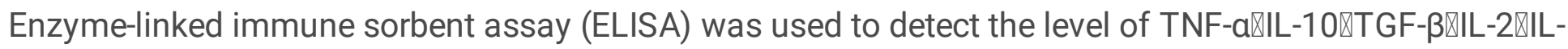
17खIL-23 in serum of each group at each time point.The specific steps were followed by the instructions of the kit.To detect spleen Tregs levels by flow cytometry:the recipient rats spleen lymphocytes were isolated and resuspended in PBS to $1 \times 10^{7} / \mathrm{ml}$ and was taken $0.1 \mathrm{ml}$ to add $0.5 \mu \mathrm{l}$ of anti-CD4 antibody and $0.625 \mu \mathrm{l}$ of anti-CD25 antibody incubating at $4^{\circ} \mathrm{C}$ for 30 minutes in the dark. After washing it with PBS $\otimes$ to incubate $1 \mathrm{ml}$ overnight at $4^{\circ} \mathrm{C}$ in the dark.It was washed with PBS and diluted to $0.1 \mathrm{ml}$ adding $5 \mu$ of antiFoxp3 antibody区incubating at $4^{\circ} \mathrm{C}$ for 2 hours in the dark.After PBS washing囚it was fixed in $4 \%$ paraformaldehyde with waiting for on-board detection. The changes of $\mathrm{CD} 4^{+}, \mathrm{CD} 25^{+}, \mathrm{Foxp}^{+}$were detected by cytometry. The differences between the groups were statistically analyzed.

\section{Statistical analysis}


Data-analysis and graph-production were performed by using GraphPad Prism 5.0 software.Statistical analysis was performed by using SPSS20. Data comparisons were performed between the two groups using Student's test.One-way analysis of variance(One-Way ANOVA)for 3 groups or above.P $<0.05$ was considered statistically significant.

\section{Results}

\section{Culture and identification of rat BMMSCs}

Rat BMMSCs were successfully cultured and expanded in vitro.It can be identified from the following

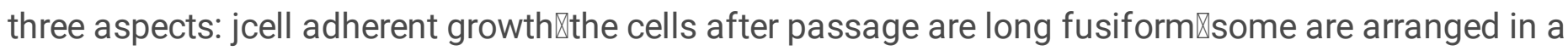
vortex or chrysanthemum with typical BMMSCs morphological features(Fig.1A).kcells can induce differentiation into adipocytes(oil red 0 staining shows red lipid droplets in the cytoplasm $\otimes$ Fig.1B) and osteoblasts(von Kossa staining showed black calcium in cells)(Fig.1C). .Detection of cell surface marker

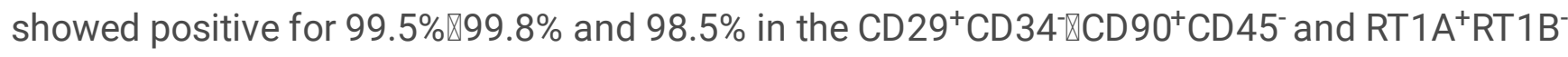
respectively (Figure $1 \mathrm{G}, \mathrm{H}, \mathrm{I})$.

\section{Recombinant adenoviral vector successfully mediated HO-1 gene into BMMSCs}

BMMSCs was infected with recombinant adenovirus expressing HO-1 gene and Ad-GFP was used as a control. The cells were observed 48 hours after infection and the positive rate of green fluorescence expression in the cells was $>80 \%$ by fluorescence microscopy (Fig.1D). Ad-HO-1/was found by immunohistochemistry.

The red-labeled HO-1 expression in the BMMSC group was significantly higher than that in the BMMSC group.In the Ad/BMMSC group (Fig.1E-F) \the expression of HO-1 gene was up-regulated and its expression level was about 5 times than the control group.These results indicate that adenovirusmediated HO-1 is overexpressed in BMMSCs successfully. The successful transfection of HO-1 laid the foundation for the next experiment.

The phenotype of BMMSCs was detected that G: the positive rate of $\mathrm{CD} 29^{+} \mathrm{CD} 34^{+} \mathrm{H}: \mathrm{CD} 90^{+} \mathrm{CD} 45^{+}$was

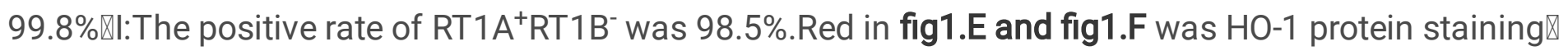
Blue is nuclear staining.

\section{Rat liver transplantation rejection model was successfully established}

In BMMSCs group,the acute rejection symptoms of the recipients were reduced to some extent and the

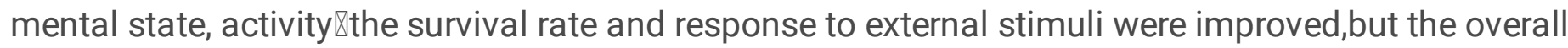
effect is still not very satisfactory.The observations in the HO-1/BMMSCs group were very encouraging that most of the recipient survived well without typical symptoms of acute rejection. During the entire

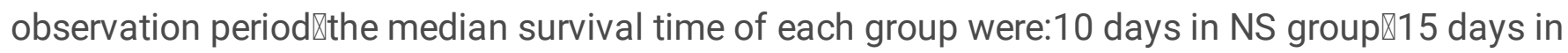
BMMSCs group and 24 days HO-1/BMMSCs group.HO-1/BMMSCs treatment further improved the 
survival rate of transplanted rats. The difference was statistically significant to compare with the BMMSCs group.

\section{Pathological manifestations of transplanted liver in each group (Fig.2)}

According to Banff criteria冈the transplanted liver in the NS group 10 days after operation showed severe rejection with mixed lymphocyte infiltration in the portal area冈inflammation and destruction in interlobular bile duct囚inflammatory cell infiltration under the interlobular vein and central vein endothelium\and hepatocyte necrosis were found. In BMMSCs group囚only mild rejection were observed.Pathological examination showed that a small amount of lymphocyte infiltration was found in the portal area囚and bile duct epithelial cell degeneration and cholangitis were rarely seen. Interlobular phlebitis and central phlebitis were not common. Compared with the other two groups, There was no obvious immune rejection pathological damage in HO-1/BMMSCs group which was close to the normal liver.

\section{BMMSCs overexpression of HO-1 induces persistent high-level expression of rat spleen Treg cells}

By flow cytometry $\mathbb{B}$ H-1/BMMSCs treatment significantly increased the expression level of $\mathrm{CD} 4^{+}, \mathrm{CD} 25^{+}, \mathrm{Foxp}^{+}{ }^{+}$in Tregs which peaked at 7 th day and fell at 10 th day(Fig.3A). Tregs ratio in BMMSCs and NS group were presented in Fig.3B.The Tregs levels in the HO-1/BMMSCs group showed a rapid and steady increase and remained high level at 10th day.Statistical analysis showed that the HO-1/BMMSCs group further increased Tregs expression compared with the BMMSCs group,especially on 10th day. The difference was statistically significant (Fig.3B).

\section{Overexpression of HO-1 enhances the regulation of cytokines in rat serum by BMMSCs}

ELISA results showed that serum IL-10 and TGF- $\beta$ in HO-1/BMMSCs group were associated with antiinflammatory or Tregs differentiation. Cytokine expression levels were further elevated compared to the control group. The increase of IL-2, TNF- $\alpha$ and IFN- $y$ in cytokines related to pro-inflammatory or Th17 differentiation was significantly smaller than that in BMMSCs group, only a slight increase, IL-6, IL-17 and IL-23 decreased or decreased. Small fluctuations, rather than the first decline in the BMMSCs group, and the overall level is significantly lower than the BMMSCs group (Fig4).The difference was statistically significant.

\section{Overexpression of HO-1 enhanced the inhibition of NK cell activity by BMMSCs Although BMMSCs} treatment significantly inhibited NK cell activity in the BMMSCs group, NK cell activity still showed a significant uptrend at 7 and 10 days. The activity of NK cells in the HO-1/BMMSCs group did not significantly increase during the observation period,but only fluctuated around normal levels. Statistical analysis showed that NK cell activity was further reduced to near normal range in the HO-1/BMMSCs group, and the difference was statistically significant(Fig5).

\section{Discussion}


Despite the advent and development of new immunosuppressants,clinical liver transplant rejection has been well controlledabut the incidence of rejection is still high. It has been reported that the incidence of acute and chronic rejection after liver transplantation can reach $90 \%$ and $30 \%-50 \%{ }^{[11]}$. The long-term use of immunosuppressive agents increases the risk of adverse reactions such as hypertension $\llbracket$ nephrotoxicity $\llbracket$ neurotoxicity $\llbracket$ postoperative tumors $\square$ and metabolic diseases ${ }^{[12]}$. Therefore $\llbracket i t$ is particularly necessary to find a new immunosuppressive strategy with few adverse reactions.

Because of its low immunogenicity,low antigen-presenting ability and unique immunomodulatory effects $\square m e s e n c h y m a l$ stem cells (MSCs) can affect the immune system such as T cells $\square B$ cells $\square N K$ cells $\square$ Monocytes and dendritic cells in vivo and in vitro ${ }^{[13-15]}$ thereby evading immune recognition and suppressing immune responses ${ }^{[16]}$. At presentıMSCs has been applied to almost all basic research of organ transplantation $\square$ and it has been found that MSCs transplanted in vivo have the characteristics of homing. Under the influence of various factors $\square$ exogenous or autologous MSCs can be directly migrated and colonized in targeted tissues ${ }^{[17-18]}$. It has also been shown to play an important role in reducing transplant immunity and inducing immune tolerance in organ transplantation ${ }^{[19]}$.

MSCs can migrate to the inflammatory colon and exert anti-inflammatory and immune-regulating effects ${ }^{[20]}$,but its intestinal colonization rate is extremely low $\square$ and MSCs is considered to be at least partially therapeutic by paracrine.At the same time, due to ischemialhypoxia and inflammatory reactions of local tissues $\square$ the survival rate after MSCs transplantation is extremely low, and the number of cells surviving 4 days after transplantation is significantly decreased while less than $1 \%$ of cells survive more

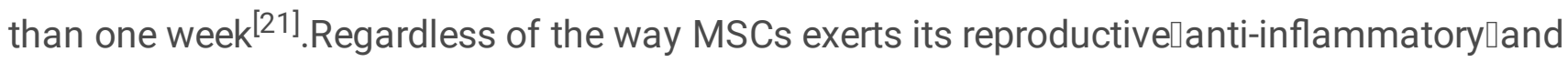
immunomodulatory effects $\square$ its effects are severely limited by low survival in vivo. Our study found that BMMSCs alleviated acute rejection in liver-transplanted rats $\square$ but this effect is limited in duration and is generally significant within 7 days and greatly weakened after 7 days.In the BMMSCs treatment group $[$ pathological examination at 10 day showed that the tissue structure was severely damaged, a large number of inflammatory cells infiltrated and apoptotic cells increased significantly which is moderate to severe rejection.Although the rejection score was lower than that of the NS group $\square$ the survival and pathological changes of the recipient mice were significantly worse than before.The various tests results suggest that BMMSCs group could not maintain a good trend in inhibiting NK cell activity $\square$ regulating cytokine expression level and inducing Tregs production within one week.

HO-1 is an inducible form of $\mathrm{HO}$, a cytoprotective enzyme that protects cells by exerting antiinflammatory,anti-apoptotic,anti-oxidative stress and anti-ischemia/reperfusion injury effects ${ }^{[22-23]}$. The study found that HO-1 also has protective and repaired effects on intestinal damage caused by various causes which main mechanisms include anti-oxidation!anti-inflammatory $\square$ anti-apoptosis and regulation of microcirculation ${ }^{[24-25]}$.In addition, $\mathrm{HO}-1$ has a regulatory effect on MSCs which can attenuate MSCs apoptosis under hypoxia and oxidative stress ${ }^{[26]}$. However, in the study of organ transplantation,HO-1 also has the effect of reducing rejection, prolonging graft survival time and even inducing graft immune tolerance ${ }^{[27-28]}$. In this study $[\mathrm{HO}-1$ was genetically engineered to modify BMMSCs.On the one hand,it is 
hoped that the cytoprotective enzyme can improve the survival rate of BMMSCs in liver-transplanted rats. On the other hand,it is hoped that both $\mathrm{HO}-1$ and BMMSCs can be immunized to superimpose inhibition effects to exert a stronger immunosuppressive effect!thereby to control liver transplant rejection better. It was found that NK cells were one of the effector cells involved in liver transplant rejection. When treated with untreated BMMSCs,the activity of NK cells was significantly decreased, but it still showed an upward trend. After treatment with gene-transfected cells(HO-1 was used),the activity of NK cells was inhibited within the normal range and did not increase significantly. We speculate that this is because BMMSCs prolonged the survival time in vivo and exerted a more sustained immunosuppressive effect. It may also be associated with immune regulation of HO-1 over-expression.

In organ transplantation,graft rejection is a T-cell-mediated immune response to donor antigens.Th10Th2םTh17ロTregs and other $\mathrm{CD} 4^{+}$Th cells play different immune effects. The ratio of Th1/Th2 and Th17/Treg play an important role in regulating T-cell immune response ${ }^{[29]}$.Th1 cells mainly

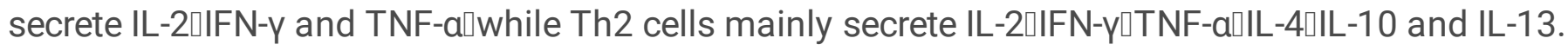
Th1/Th2 ratio and related cytokines are often used to explain immune related phenomena in organ transplantation. Th17 cells are characterized by the secretion of cytokines IL-17,IL-21and IL-22. Their differentiation requires the participation of TGF-beta and IL-6, and the stabilization of their phenotype requires IL-23, TNF-alpha and IL-1 beta ${ }^{[30-32]}$. Tregs can produce anti-inflammatory effect and promote self-tolerance by secreting IL-10 and TGF- $\beta$. Th 17 cells and their cytokines IL-6, IL-17 and IL-23 may mediate transplantation rejection ${ }^{[33]}$. Tregs may prevent rejection and even induce to maintain immune tolerance ${ }^{[34]}$.The immunoregulation of MSCs is related to the amplification of Tregs. In some studies $\square$ MSCs can induce Foxp3 ${ }^{+}$and Tregs-dependent tolerance ${ }^{[35]}$.

In this research,inflammation and Th differentiation-related cytokines have significant changes under the treatment of BMMSCs and HO-1/BMMSCs.Specifically, the concentrations of IL-10 and TGF- $\beta$ increased significantly,while the concentrations of IL-2,IL-6,IL-17,IL-23,TNF- $\alpha$ and IFN- $\gamma$ decreased significantly.The difference is that compared with BMMSCs group $\mathrm{H}$ O-1/BMMSCs group increased IL-10ロTGF- $\beta$ and

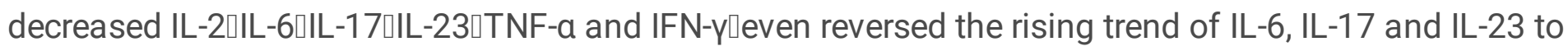
some extent. The change of Tregs is also very significant. HO-1/BMMSCs not only up-regulated Tregs expression $\square$ but also its high level expression was more stable and lasting.We speculate that these effects are related to protecting BMMSCs and enhancing its immunoregulation of $\mathrm{HO}-1$.

In summary,compared to BMMSCs alone in this study[HO-1/BMMSCs inhibition of liver transplant rejection by the BMMSCs group was stronger and longer lasting mainly by inhibiting immune cells such as NK cells, Treg cells and cytokines work.

\section{Declarations}

Author Contribution statement

Peng Li contributed to the conception of the study and conceived and designed the experiments 
Yuyi Zhang performed the experiments and contributed significantly to analysis

Qiongxia Li performed the data analyses and wrote the manuscript

Yubo Zhang helped perform the analysis with constructive discussions.

\section{References}

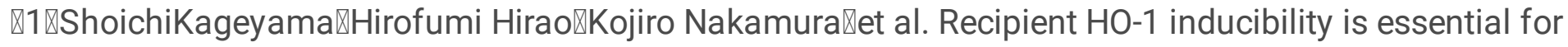

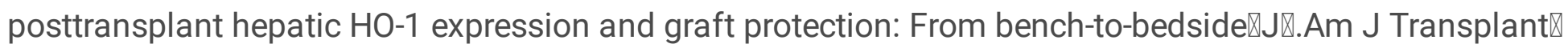
2019囚19(2):356-367.

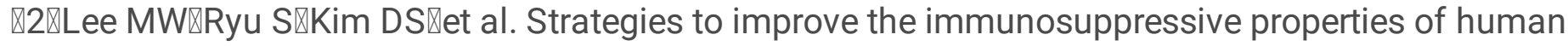

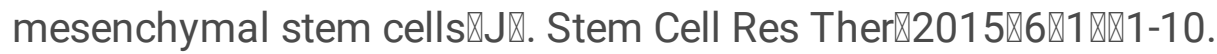

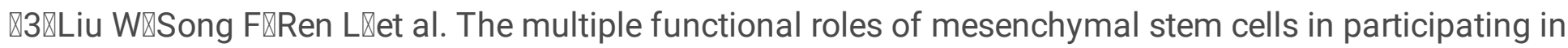

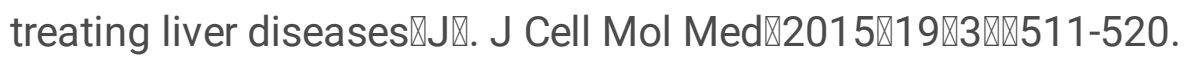

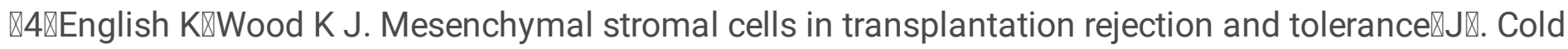

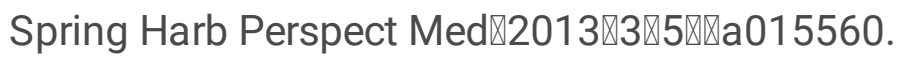

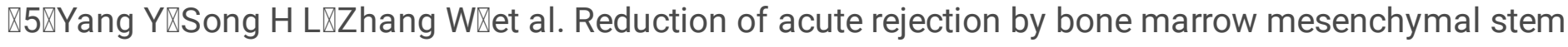

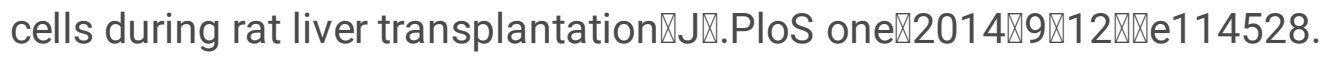

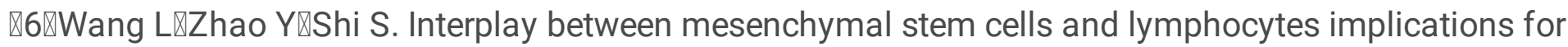

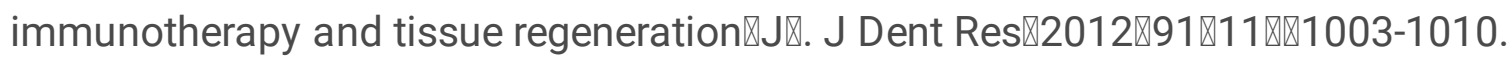

\7هHedblom A, Hejazi SM, Canesin G, et al. Heme detoxification by heme oxygenase-1 reinstates

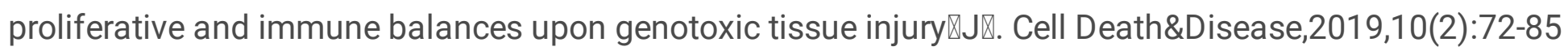

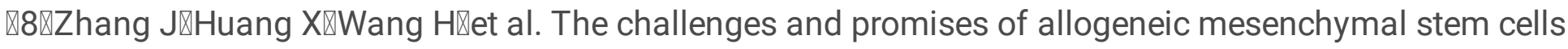

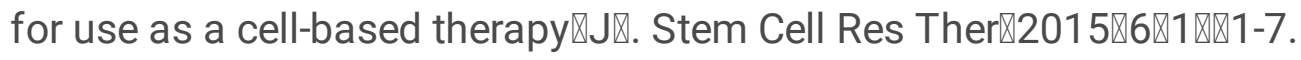

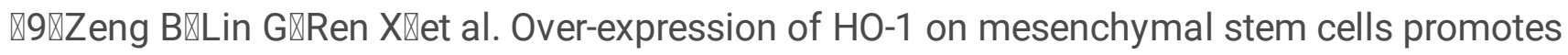

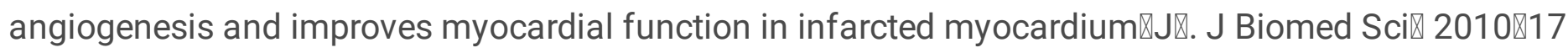
$凶 1 \otimes \square 80-88$.

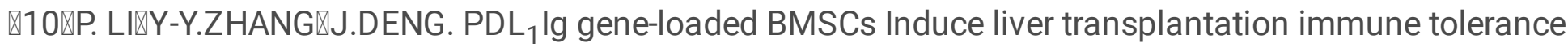

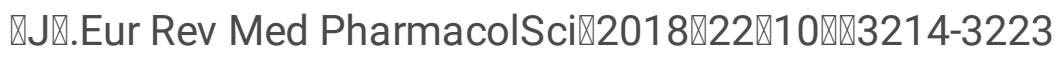

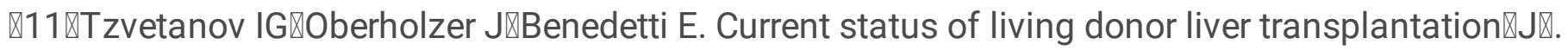
CurrOpin Organ Transplant $₫ 2010 \otimes 15 \rrbracket 3 \otimes \bigotimes 346-348$. 


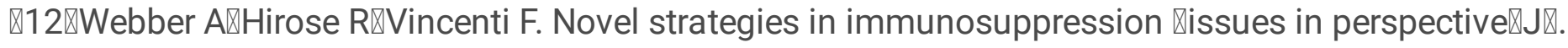
Transplantation $₫ 2011 \otimes 91 \otimes 10 \otimes \square 1057-1064$.

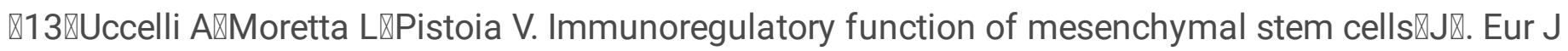

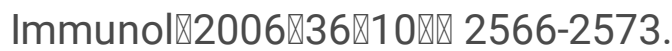

\14『Le Blanc K囚Tammik L囚Sundberg B囚et al. Mesenchymal stem cells inhibit and stimulate mixed lymphocyte cultures andmitogenic responses independently of the major histocompatibility complex区J区. Scand J Immunol $₫ 2003 \otimes 57 \otimes 1 \otimes \otimes 11-20$.

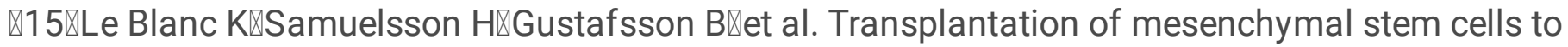

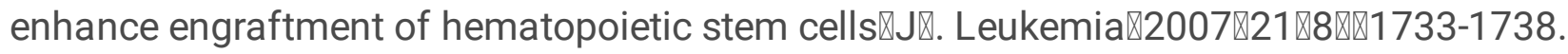

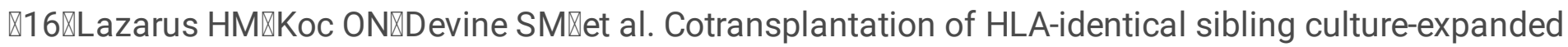

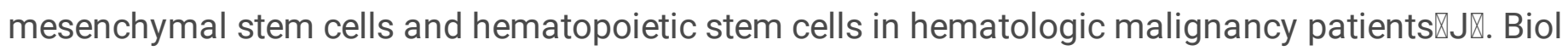
Blood Marrow Transplant $₫ 2005 \rrbracket 11 \rrbracket 58 \rrbracket 389-398$.

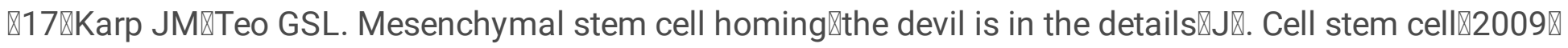
4囚3ष囚206-216.

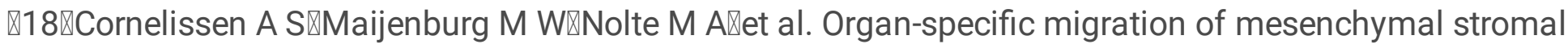

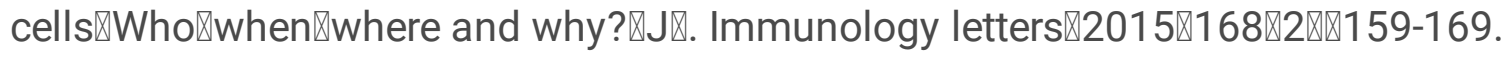

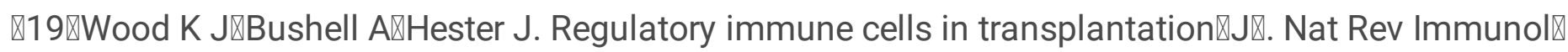

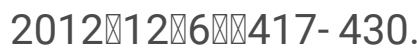

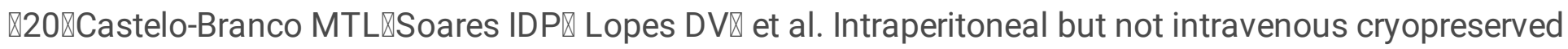
mesenchymal stromal cells home to the inflamed colon and ameliorate experimental colitis $\mathbb{J} \mathbb{\text { V}}$. PLoS One $2012 \rrbracket 7 \rrbracket 3 \rrbracket \bigotimes e 33360$.

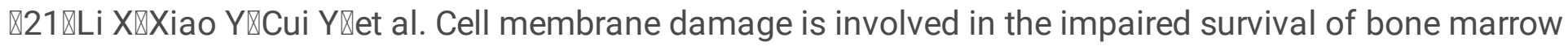

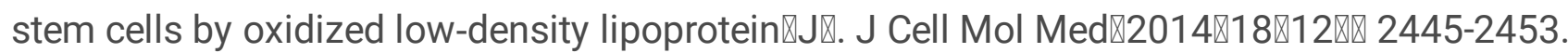

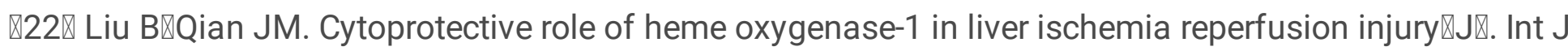

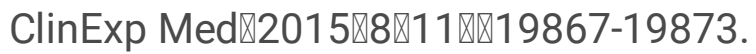

$\triangle 23 \llbracket$ Lawal AO『Marnewick JL『Ellis EM. Heme oxygenase-1 attenuates cadmium-induced mitochondrial-

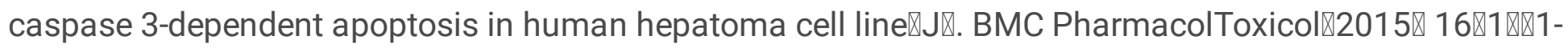
13.

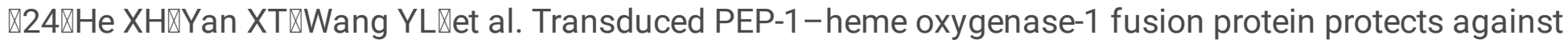

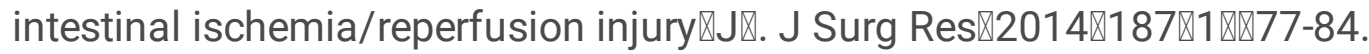




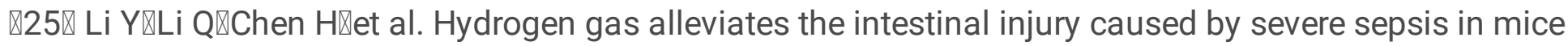

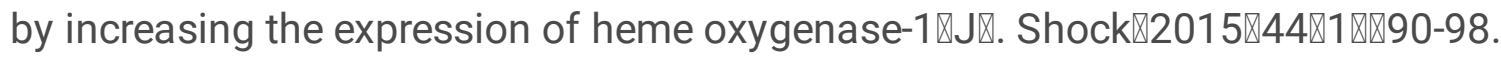

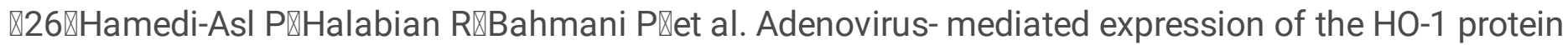

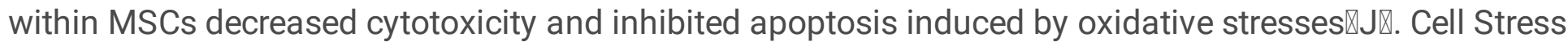
Chaperones $₫ 2012 \otimes 17 \rrbracket 2 \otimes \bigotimes 181-190$.

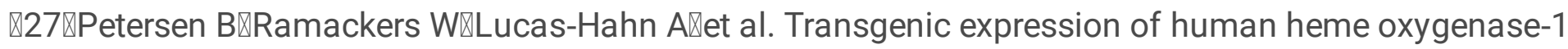

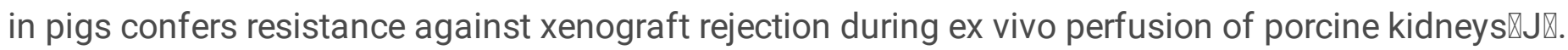
Xenotransplantation $₫ 2011 \otimes 18 \otimes 6 \mathbb{6} \otimes 355-368$.

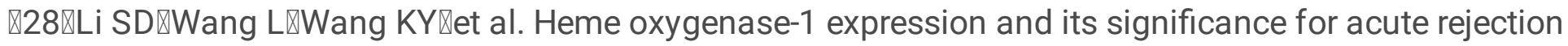

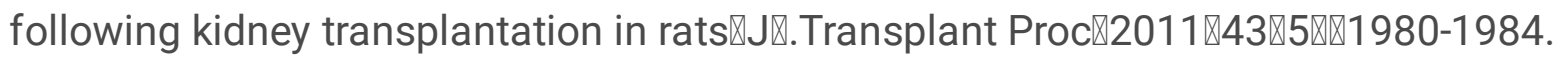

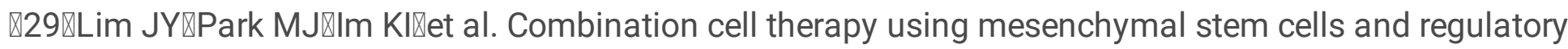
T-cells provides a synergistic immunomodulatory effect associated with reciprocal regulation of $\mathrm{TH} 1 / \mathrm{TH} 2$ and th17/treg cells in a murine acute graft- versus-host disease mode『l J囚. Cell transplantation $₫ 2014 \llbracket 23 \rrbracket 6 \rrbracket \rrbracket 703-714$.

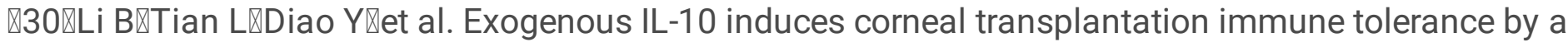
mechanism associated with the altered Th1/Th2 cytokine ratio and the increased expression of TGF- $\beta$

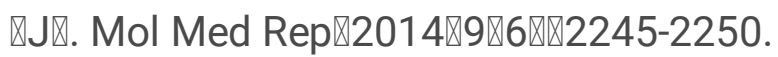

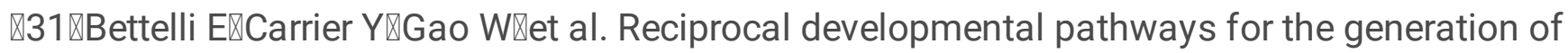

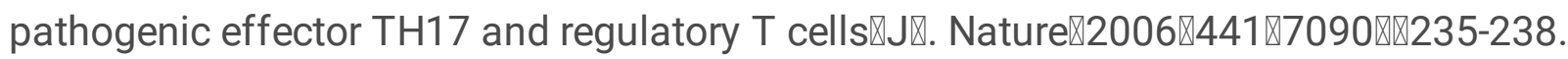

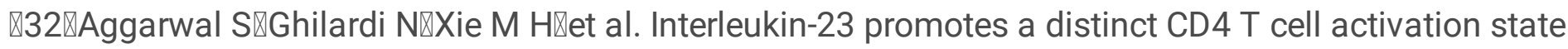

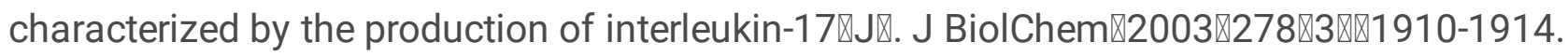

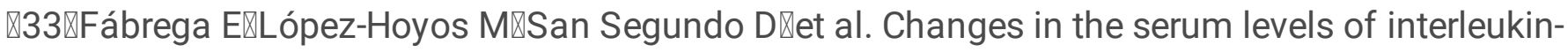

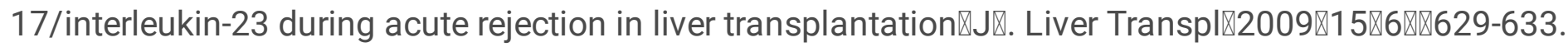

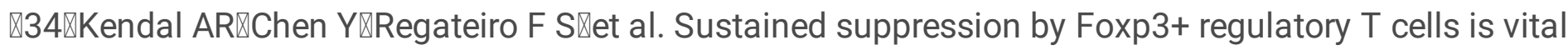

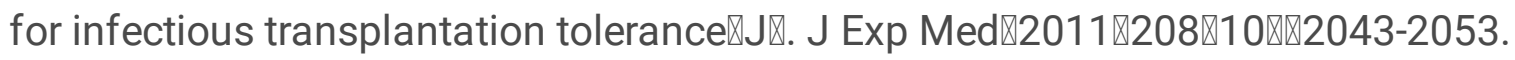

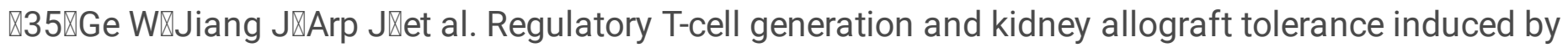
mesenchymal stem cells associated with indoleamine $2 \varangle 3$-dioxygenase expression $₫ \mathrm{~J} \bigotimes$. Transplantation $\rrbracket$ 2010ه90®12ه $1312-1320$.

\section{Figures}


A

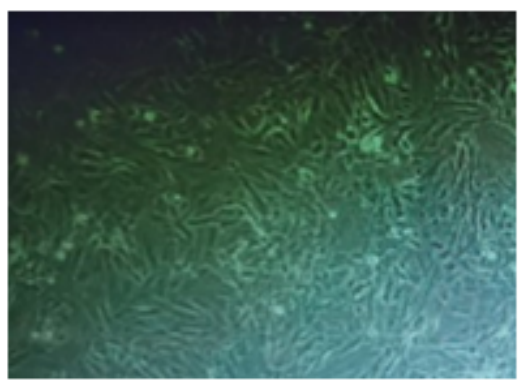

D

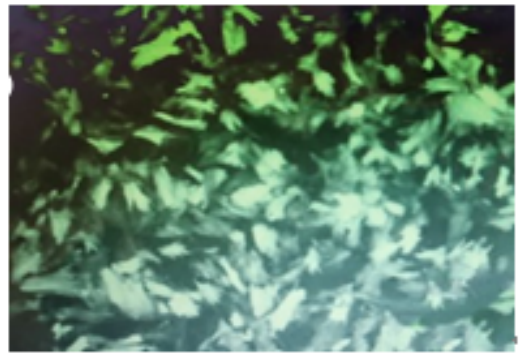

$\mathrm{G}^{-}$

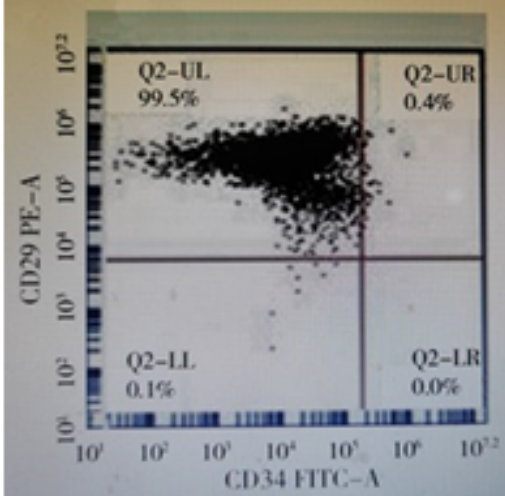

$\mathrm{B}$
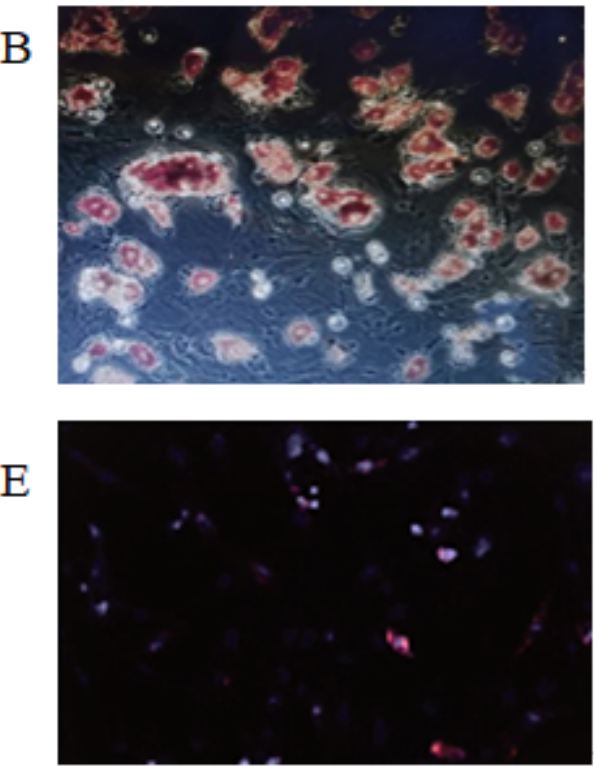

$\mathrm{H}$

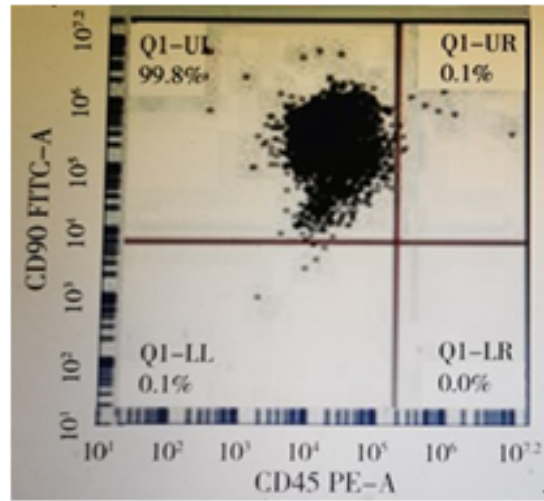

$\mathrm{C}$

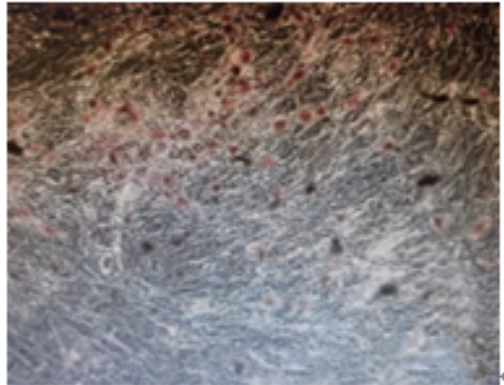

F

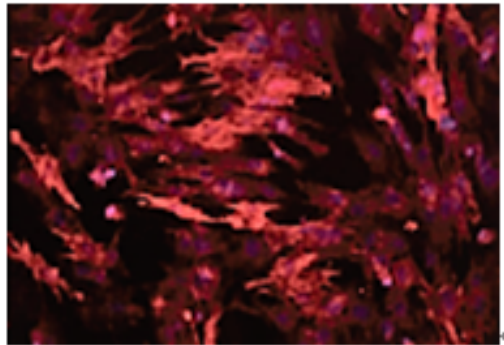

I

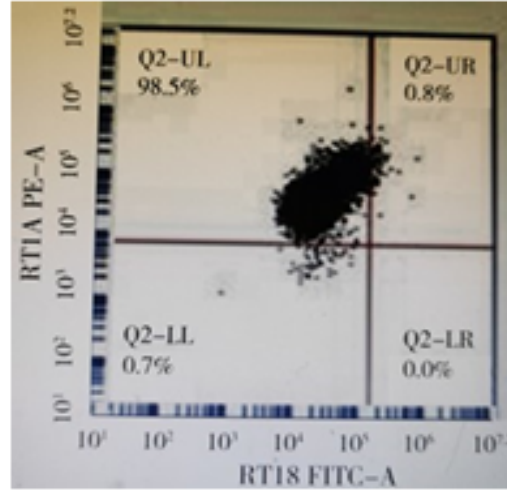

\section{Figure 1}

Morphology, characteristics, phenotype and HO-1 transfection of BMMSCs A囚The morphology of BMMSCs in the third generation (100x)『B:adipogenic test of BMMSCs』C:osteogenic verification of BMMSCs (200x) $₫ \mathrm{D}$ :The dark field of BMMSCs transfected with HO-1(100x) $₫ \mathrm{E}: \mathrm{HO}-1$ expression in BMMSCs without $\mathrm{HO}-1$ transfection $\otimes \mathrm{F}: \mathrm{HO}-1$ expression in BMMSCs transfected with $\mathrm{HO}-1$. The expression level was significantly higher than that in the untransfected group(200x).

A

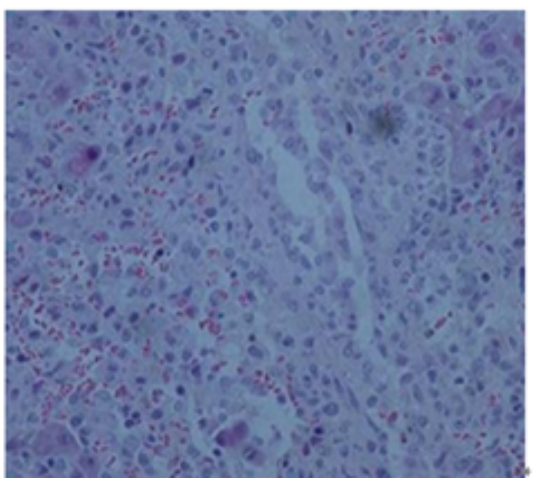

$\mathrm{B}$

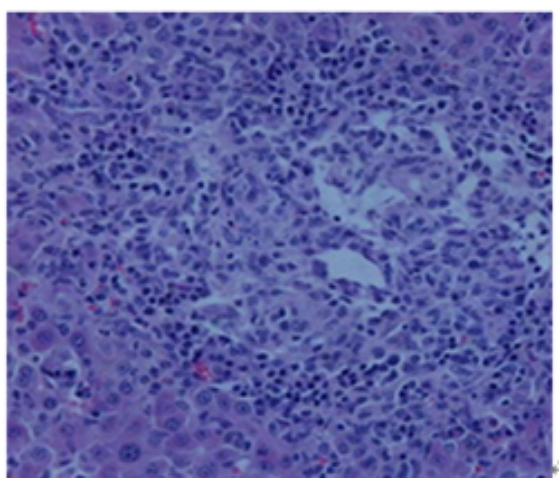

$\mathrm{C}$

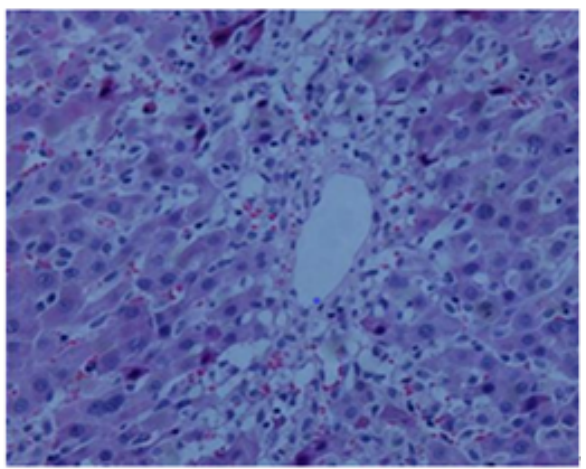

Figure 2

Pathological findings of LT in each group (100x) A: NS group B囚BMMSCs group C『HO-1/BMMSCs 

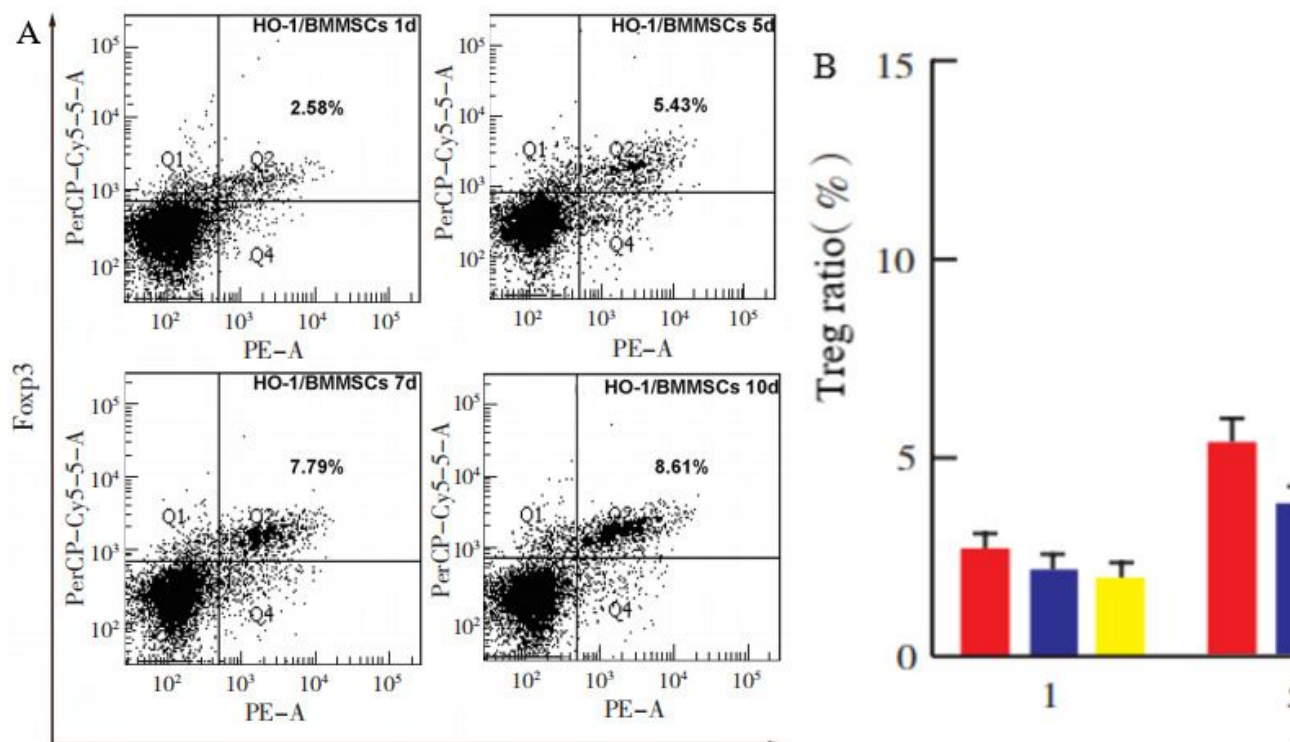

CD25

\begin{abstract}
5
\end{abstract} days after transplant
HO-1/BMMSCs

BMMSCs

NS

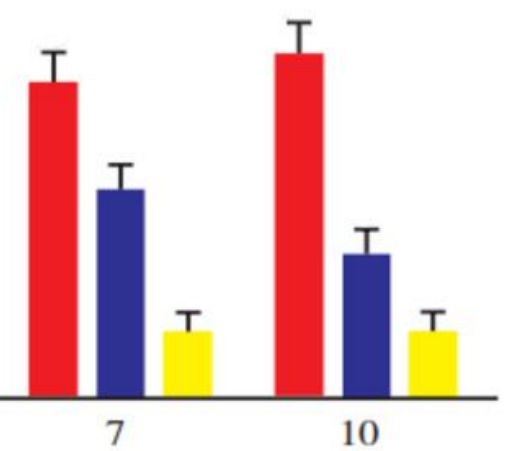

10

\section{Figure 3}

The level of Treg in recipient spleen A. Scatter plot in Flow cytometry of Tregs in spleen of HO-1/BMMSCs group at different time points after operation. B. Tregs ratio of rats in each group(compared with NS group $\triangle \mathrm{P}<0.05 \llbracket$ compared with $B M M S C$ s group $\triangle \mathrm{P}<0.05$ ) 

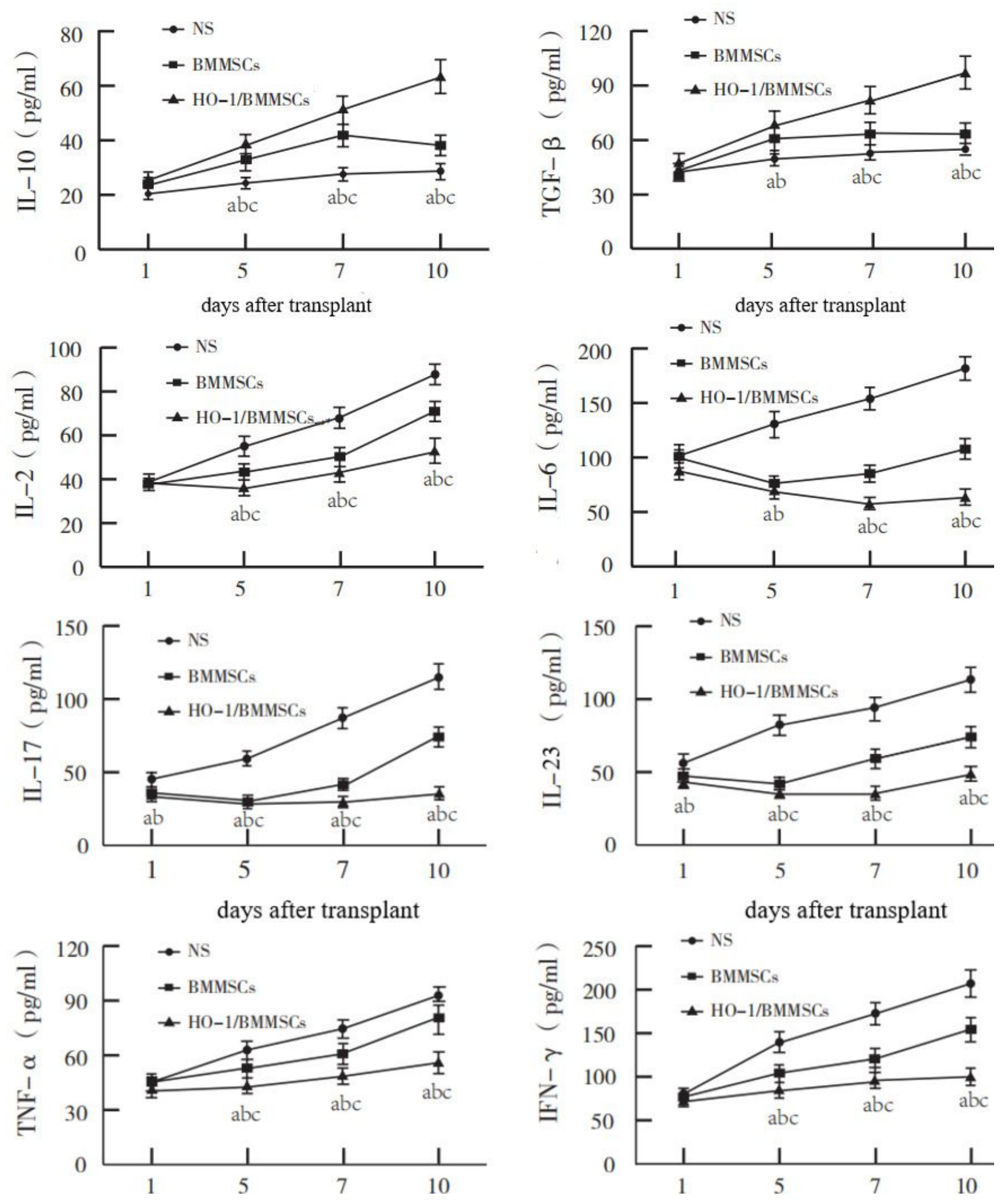

\section{Figure 4}

Each cytokines levels in serum (compared with NS group, P®0.05『Compared with BMMSCs group and Compared with HO-1/BMMSCs, P₫0.05) 


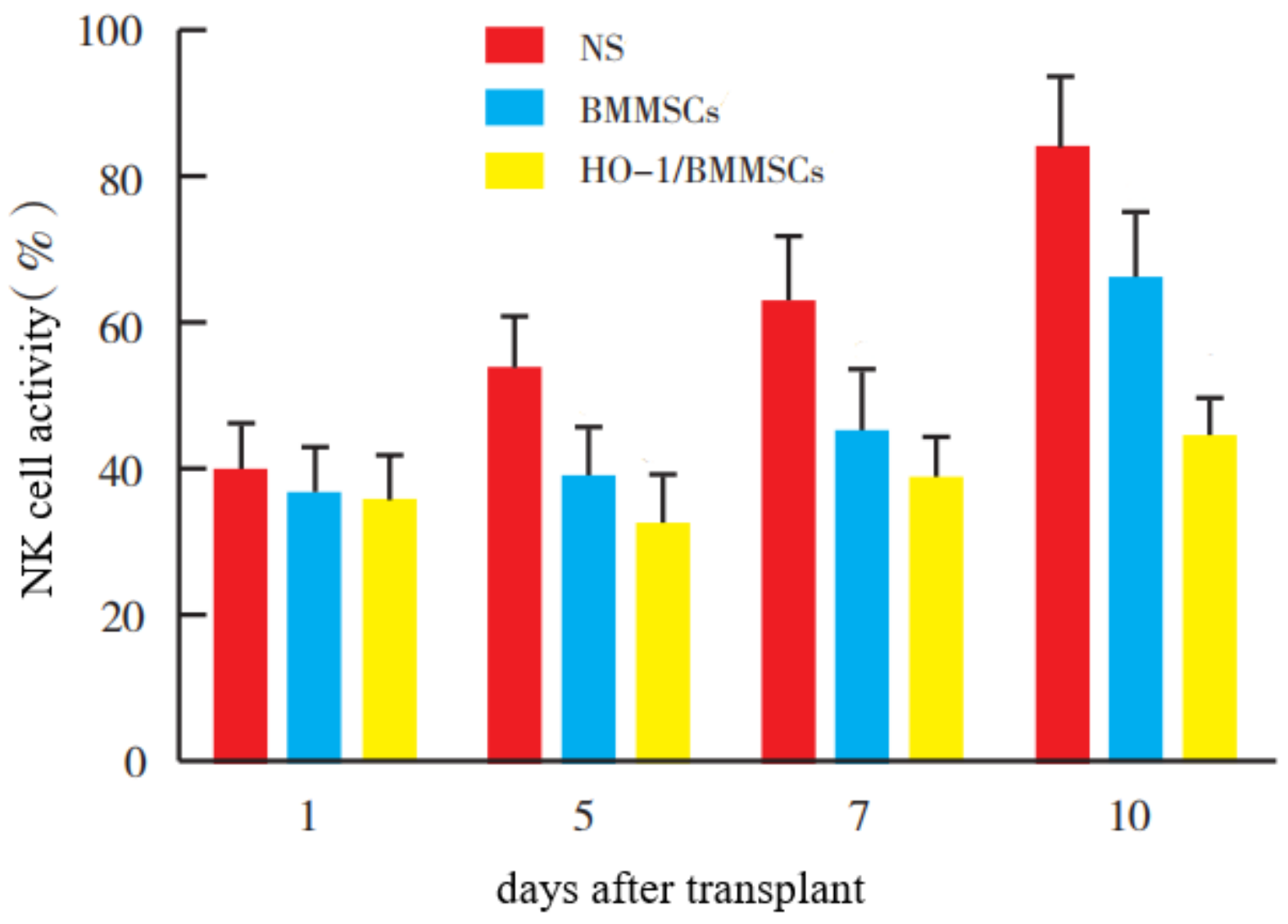

Figure 5

NK cell activity Compared with NS group, $\mathrm{P}<0.05$,compared with BMMSCs group, $\mathrm{P}<0.05$ 